\title{
Building Management System in WebBIM Environment
}

\author{
Danylo Shkundalov, Tatjana Vilutienë \\ Department of Construction Management and Real Estate, Faculty of Civil Engineering, \\ Vilnius Gediminas Technical University, Vilnius, Lithuania
}

Received 4 February 2020; accepted 31 March 2020

\begin{abstract}
Geographical information systems (GIS) and Building information modelling (BIM) provide digital representation of building, its elements and environment. BIM focuses on micro-level representation of buildings and its elements, and GIS provide macro-level representation of the external elements of environment. Their combination can provide a comprehensive view of a built environment based on integrated data. There is an opportunity to make BIM and GIS unity not only to combine them in a single project but also to make a fully merged environment with difficult associations that extend the capabilities of both. Paper presents method that allows visualization and processing the BIM model in the web browser, external processing and analyzing, easy sharing and visualization, linking the 3D model and attribute information, working with the BIM model and GIS data, digital City mapping and etc. The developed method creates rich future for full BIM software products with all manner of functions and tools that will work through the Internet without installations, distributives, additional programs. The introduced technique can be used as a new part for BIM execution planning.
\end{abstract}

Keywords: 3D model, GIS, BIM, WEB, WebGL, coordinates, conversion.

\section{Introduction}

The Geographic information system (GIS) and Building information modelling (BIM) collaboration can increase the effectivity of both environments such as using GIS data for representing a large scale information and BIM models to present a smaller such as buildings or even indoor objects (Azhar, 2011). Although, many projects that allow visualization of the BIM models representations exist, which are highly useful in the project designing process (Zhou et al., 2018; Feng et al., 2012; El-Mekawy et al., 2011; Liu et al., 2016), the collaboration of BIM and GIS inside the Web environment faces a big amount of issues because this methodology is pretty new and just started developing.

BIM and GIS collaboration is one of the widely discussed topics nowadays in the civil engineering field that can bring many benefits for a building life cycle. The review made by Liu et al. (2017) revealed that there are still many obstacles and challenges to the achievement of BIM and GIS integration, though, interoperability of those environments can be proved by the big amount of the different scientific articles and projects around the world (Liu et al., 2017). Construction projects contain a big amount of project related data that should be stored and processed in the management system with the proper document structure. Such a system can bring many benefits such as analysis and extra functions apart from just file management; however, such system should take care about all sides of the project such as model files, planning, analysis and their results, the collaboration of the participants, data transfer, documents actualization, etc. Pauwels et al. (2017) and Wong et al. (2018) made an analysis of the existing developments and concluded that no exchange specifications has been developed and integrated that has results in lack of solutions to define a proper way of collaboration. Ma and Ren (2017) revealed that usage of unified models based on IFC and CityGML standards could bring a benefit in solving some issues. As an effort to organize the wellstructured exchange and provides the possibility to access the project from any place of the world for all project participants the Web technology can be used. As Web-based Graphics Library (WebGL) environment brings the opportunity to visualize the 3D models in the web environment then it can be used to process BIM models as well.

The analysis of the literature revealed that there is no universal conversion method between BIM and GIS. The compatibility of the Web, BIM and GIS environments coordinate systems is one of the major sticking points in collaboration of these environments (Arroyo Ohori et al., 2018). Some efforts are being made in this direction, for example, $\mathrm{Xu}$ et al. (2020) presented a good solution for the BIM model representation in a GIS environment with the aim of quick rendering in the WebGL environment where Cesium library has been used for the web visualization and Autodesk Revit for the IFC file export. The main advantage of proposed solution is in using 3D tiles for model

*Corresponding author. E-mail: tatjana.vilutiene@vgtu.lt

\section{Copyright (C) 2020 The Author(s). Published by VGTU Press}

This is an Open Access article distributed under the terms of the Creative Commons Attribution License (http://creativecommons.org/licenses/by/4.0/), which permits unrestricted use, distribution, and reproduction in any medium, provided the original author and source are credited. 
representation that are generated on the model conversion step. Such tiles generation allows to visualize large scale models with big amount of objects, however it leads to strict coordinate dependency that, according to presented article, are calculated during IFC to OBJ conversion step and no web-based solution for BIM model representation and 3D tiles relocation is presented.

The existing methods generally perform the conversion process through a third-party file format such as OBJ, but these files cannot hold the semantic information in the BIM file. Taking into account the shortcomings of recently proposed solutions, this article proposes the approach how the BIM model representation coordinates can be specified and changed inside the GIS environment within the help of BIM management system based in the web environment.

\section{Proposed model for web-based BIM management system coordinate specification}

The main aim of the proposed approach is to develop the easy and useful way to specify the coordinates of the BIM model inside the web environment as geodesic coordinate systems cannot be used to present the information inside the web environment by default. The main difference of this approach is that model position is not constantly defined while loading process or inside the proprietary GIS software but within the help of web-based BIM management system that allows changing the place of the BIM representation model in any time. Such functionality expends the possibilities of the architects and engineers in urban planning, conceptualization, site and solar analysis in the condition of real environment 3D mesh model and other BIM uses cases. The proposed approach is divided into following steps:

- Step 1. Development of the Web-based management system functionality.

- Step 2. Specifying or detecting the model coordinates.

- Step 3. Converting the coordinates into web supportable coordinate system.

- Step 4. Placing the model inside the GIS environment.

The steps of proposed approach described in following sections.

\subsection{Development of the Web-based management system functionality}

The management system need to have an opportunity to load the BIM representation model into the web environment and process it's objects in the way as they would be processed inside the BIM environment. For this purpose, the GIS environment need to be used and the best solution for this is to use the web-based map.

The management system need to have the opportunity to load and process the BIM model inside the web environment. The good overview of the web-based BIM platforms was done by Shkundalov and Vilutienè (2019a) with the comparison of their possibilities. From this article can be seen that all of the presented platforms have opportunity to connect GIS data to the platform, however, not all of them can process and manipulate with the BIM models. Moreover, the in-model objects do not have a coordinate system recalculation as well as no object relations that means that they can be considered as a group of objects but not as a single complex object. In other words, the BIM model can be placed and visualized on the map but not objects presented in the model therefore the positioning of the objects by the geodesic coordinates is not possible.

To allow the web-based management system to specify the coordinates for the BIM model representation in the condition of web environment the system need to have needed functionality. For this purpose, the usage of web-map will be the most suitable solution because it gives the easiest way to place the object and detect its coordinates and present the result on the map layer that can be 2D or 3D mesh. To achieve this point any map can be used, for this article the mapbox library (Mapbox) is used which is based on OpenStreetMap. The advantage of this solution is that it is easy to use in the project: the map initialization code is presented in code 1 (Figure 1). The presented code creates the map layer that outputs the longitude and latitude of the marker position that can be used for the model positioning within JavaScript or php functionality.

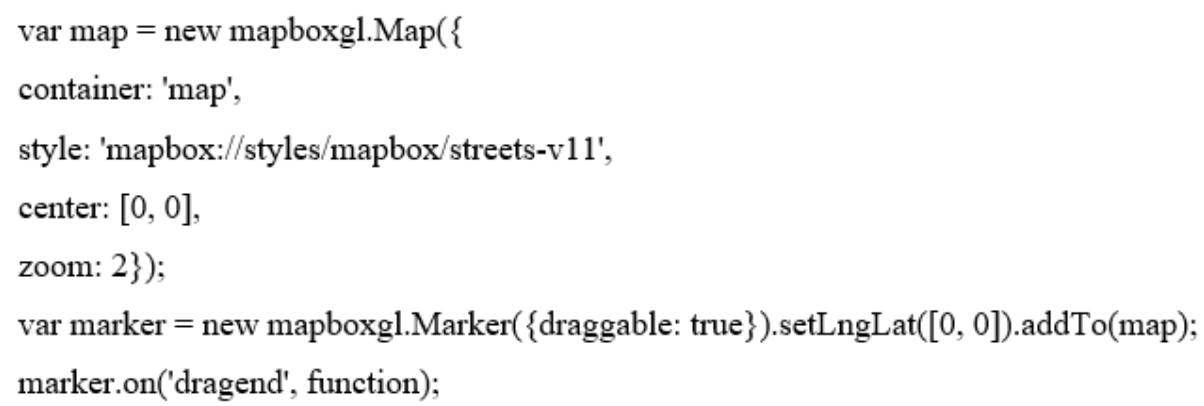

Figure 1. Code 1 - initializing the mapbox map layer with dragable marker 
The management system need to have an opportunity to save the resulting coordinates that can be done in many different ways: storing in database within link to model identificator by SQL request, stored in model-related files in the server storage or even implemented inside the BIM model representation file, however, the last variant obstruct the model updating process, therefore, it should not be used. For the aim of the article, the resulting coordinates has been saved in the model-related file as .json file type. This solution allows easy update the model position afterwards by management system with no need to change the structure of the model or connect to the database.

\subsection{Specifying or detecting the model coordinates}

WebGL does not support any of the known BIM software proprietary formats, as it only supports simple formats. To make possible to visualize information in the browser, the existing BIM model formats need to be converted into the well-known file formats such as .obj or any other web supportable file formats (Shkundalov, 2017). Web representation of BIM model is not the same as native BIM model that is used inside the proprietary software such as Autodesk or Bentley solutions because the web environment does not has the same structure and cannot process BIM models in a native way. Moreover, the attributive information should be presented and linked with objects inside the model to give an opportunity to process that objects in a similar way as inside the BIM environment.

The proposed approach made by Shkundalov and Vilutienè (2019b) propose a full concept of BIM model representation attributive information processing including the object relations and rules determining, coordinate recalculation, etc.: "Due to implemented relations and rules, the objects in the BIM model can be considered not just like independent 3D objects in the WebGL environment as all object are presented by default, but as objects that are linked between each other and can act by the rules that defined to each type of the object. These rules are the main key factor that makes the difference between the usual 3D object and BIM model representation in the WebGL environment". The proposed approach allows determining the in-model objects coordinates and convert them to be used inside the web environment. In case the BIM model representation has the coordinates that allows to place the model inside the GIS environment than the proposed approach allows to recalculate the coordinates in respect of new coordinate system.

\subsection{Converting the coordinates into Web supportable coordinate system}

The model can be placed in two ways: by the map tools or by using the geodesic coordinates. In case of map tool usage the user is able to drag the point around the map and specify the place where the model should be placed. The output of the map source is presented as longitude and latitude in web Mercator projection such as "25.337594076107194" and "54.72161336870195" respectfully.

In case of using the geodesic coordinates, they need to be converted into web coordinate system to give possibility for margin the model with the map source. For this article the WGS84 (EPSG:4326) coordinate system has been used. The resulting coordinates of the conversion need to be presented as unit vector of Mercator projection (EPSG:900913) coordinate system as web environment does not support other coordinate systems. The conversion can be done by using the formula (1) presented below within the JavaScript functionality (Conversion of ...), where $\lambda-$ longitude, $\varphi-$ latitude.

$$
\left\{\begin{array}{c}
x=\lambda * \frac{20037508.34}{180} \\
y=\frac{\log \left(\tan \left((90+\varphi) * \frac{\pi}{360}\right)\right)}{\frac{\pi}{180}} * \frac{20037508.34}{180}
\end{array}\right.
$$

In case the map use metric system then the Mercator projection coordinates can be converted by the formula (2).

$$
\left\{\begin{array}{c}
\mathbf{x}_{\mathbf{m}}=\frac{\mathbf{x}+20037508.34}{2 * 20037508.34} \\
\mathbf{y}_{\mathbf{m}}=1-\frac{\mathbf{y}+20037508.34}{2 * 20037508.34}
\end{array}\right.
$$

\subsection{Placing the model inside the GIS environment}

This part of the BIM model representation positioning process is the smallest but the most important because the future model analysis will be performed based on the model position in condition of the map layers that is going to have a big influence to the results specially if the $3 \mathrm{D}$ mesh model of the real environment is going to be used. The coordinates 
received from the management system now can be used to define the position of the model. As at this point the proposed approach already brings coordinates of the web Mercator projection coordinate system they can be used straight ahead to the map layer. In case of mapbox to add the model to the map layer the following code is enough (Figure 2).

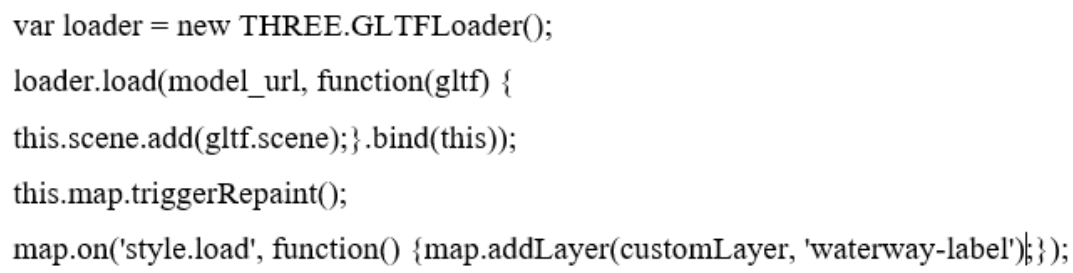

Figure 2. Code 2 - adding model to the mapbox map layer

The mapbox uses the Three.js WebGL library to process and visualize the data in the web browser. All process of three.js scene creation and required related objects has been described by the Shkundalov (2017). After the model is placed inside the web environment it's position can be granted from the BIM management system. For this article the coordinates of the model has been stored in .json file on the server and applied to the model. The resulting scene with the loaded BIM representation model into web environment is presented in Figure 3. The map layer is presented by OpenStreetMap, the JavaScript functionality has been developed based on by Three.js and mapbox libraries, BIM model representation has been done from Autodesk Revit.

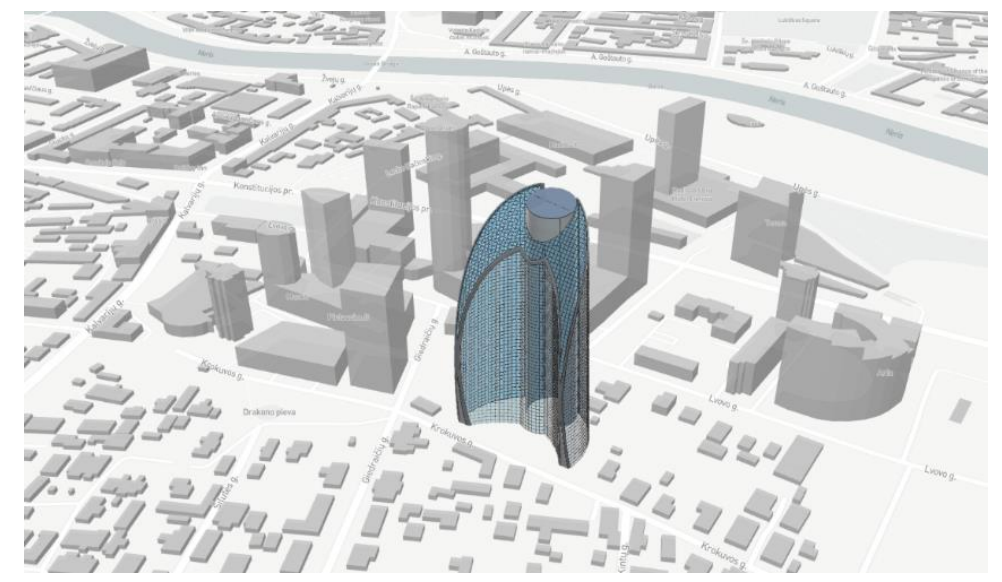

Figure 3. The positioning of the web environment to represent BIM model

\section{Discussions and further research}

Mainly BIM is used for vertical construction such as buildings and all its components and systems, stadiums, power stations. This is mainly due to the fact that vertical construction projects are using two coordinate systems in the same time: the in-project linear coordinate system that is needed to construct all parts of the project in one software environment; and single geodesic coordinate system that is used to place the project in the real world position for the further construction phases. The proposed approach allows us to recalculate the geodesic coordinate system but does not perform any in-project coordinate system changes; therefore, this approach is not suitable for the horizontal construction at the existing phase. Horizontal (transportation) and vertical (buildings) construction has different operations, components and techniques from planning through construction, to operation and maintenance, as it has been mentioned by Dell'Acqua et al. (2018). Horizontal construction additionally relays on GIS data due to the length of the construction objects such as railway, roads, tunnels, etc. While construction of such long objects more than one coordinate system needs to be considered as an object can intersect different geodesic projections, therefore multiple stations and alignment curves are used as references. The development and implementation of the tools and technics for recalculation of coordinates for the horizontal projects inside the Web environment is next step in the development of Web-based BIM management system as such research will allow to place and process highlighted above objects in a more accurate way and expend the collaboration of the BIM, GIS, and Web environments. 


\section{Conclusions}

The proposed approach to use the web-based BIM management system to specify the BIM model representation position in the GIS environment brings two important features to the BIM project management process:

1. flexibility of the model position that is useful on planning stage of the BIM life cycle;

2. opportunity to convert into web Mercator projection coordinate system any geodesic coordinate system regardless the software where the model has been created, because the model positioning is going to be defined on the server side and not inside the proprietary software.

Moreover, in case the model is not defined in exact coordinates then they can be easily applied with the purpose to perform different analysis such as logistic, safety analysis, decision-making, urban planning, conceptualization, etc.

In case the BIM model represents a complex group of objects or structures then each of them can inherit the coordinates of the BIM model and calculate their coordinates not just inside the web environment coordinate system but in the geodetic coordinate system as well that can be useful on the construction site.

\section{References}

Arroyo Ohori, K., Diakité, A., Krijnen, T., Ledoux, H., \& Stoter, J. (2018). Processing BIM and GIS models in practice: experiences and recommendations from a GeoBIM project in the Netherlands. ISPRS International Journal of Geo-Information, 7(8), 311. https://doi.org/10.3390/ijgi7080311

Azhar, S. (2011). Building information modeling (BIM): Trends, benefits, risks, and challenges for the AEC industry. Leadership and Management in Engineering, 11(3), 241-252. https://doi.org/10.1061/(ASCE)LM.1943-5630.0000127

Dell'Acqua, G., Oliveira, S., \& Biancardo S. (2018). Railway-BIM: Analytical review, data standard and overall perspective. Ingegneria Ferroviaria, 73(11), 901-923.

El-Mekawy, M., Östman, A., \& Shahzad, K. (2011). Towards interoperating CityGML and IFC building models: a unified model based approach. In Advances in 3D geo-information sciences (pp. 73-93). Springer. https://doi.org/10.1007/978-3-642-12670-3_5

Feng, L., Wang, C., Li, C., \& Li, Z. (2011, December). A research for 3D WebGIS based on WebGL. In Proceedings of 2011 International Conference on Computer Science and Network Technology (pp. 348-351). IEEE. https://doi.org/10.1109/ICCSNT.2011.6181973

GitHub Gist. (2019). Conversion of the coordinates. https://gist.github.com/springmeyer/871897

Liu, X., Wang, X., Wright, G., Cheng, J., Li, X., \& Liu, R. (2017). A state-of-the-art review on the integration of Building Information Modeling (BIM) and Geographic Information System (GIS). ISPRS International Journal of Geo-Information, 6(2), 53. https://doi.org/10.3390/ijgi6020053

Liu, X., Xie, N., Tang, K., \& Jia, J. (2016). Lightweighting for Web3D visualization of large-scale BIM scenes in real-time. Graphical Models, 88, 40-56. https://doi.org/10.1016/j.gmod.2016.06.001

Mapbox. (2019). Documentation. Examples, tutorials, and API references to help you start building with Mapbox. https://docs.mapbox.com/

Ma, Z., \& Ren, Y. (2017). Integrated application of BIM and GIS: An overview. Procedia Engineering, 196, 1072-1079. https://doi.org/10.1016/j.proeng.2017.08.064

Pauwels, P., Zhang, S., \& Lee, Y. C. (2017). Semantic web technologies in AEC industry: A literature overview. Automation in Construction, 73, 145-165. https://doi.org/10.1016/j.autcon.2016.10.003

Shkundalov, D. (2017, March). Development of visualization and manipulation methods for BIM and digital city models using Web graphic library. In Proceedings of the $20^{\text {th }}$ Conference for Junior Researchers "Science - Future of Lithuania": "Civil Engineering and Geodesy”. Vilnius, Lithuania. eISSN 2029-7149. Article Number: msk.2017.313.

Shkundalov, D., \& Vilutienè, T. (2019a, May). The analysis of Web technologies for BIM model processing. In $17^{\text {th }}$ International Colloquium "Sustainable Decisions in Built Environment". Vilnius, Lithuania. https://doi.org/10.3846/colloquium.2019.009

Shkundalov, D., \& Vilutienė, T. (2019b, May). A new approach for extending the possibilities of collaboration between BIM, GIS and Web environments to increase the efficiency of building space management. In Proceedings of the $13^{\text {th }}$ International Conference "Modern building materials, structures and techniques". Vilnius, Lithuania. https://doi.org/10.3846/mbmst.2019.057

Wong, J. K. W., Ge, J., \& He, S. X. (2018). Digitisation in facilities management: A literature review and future research directions. Automation in Construction, 92, 312-326. https://doi.org/10.1016/j.autcon.2018.04.006

Xu, Z., Zhang, L., Li, H., Lin, Y. H., \& Yin, S. (2020). Combining IFC and 3D tiles to create 3D visualization for building information modeling. Automation in Construction, 109, 102995. https://doi.org/10.1016/j.autcon.2019.102995

Zhou, X., Wang, J., Guo, M., \& Gao, Z. (2018). Cross-platform online visualization system for open BIM based on WebGL. Multimedia Tools and Applications, 78(20), 28575-28590. https://doi.org/10.1007/s11042-018-5820-0

\section{Notations}

Variables and functions

$\lambda$ - longitude

$\varphi$ - latitude

\author{
Abbreviations \\ BIM - Building Information Modelling \\ GIS - Geographic Information System \\ IFC - Industry Foundation Classes (International Alliance for Interoperability)
}

